Coronavirus(in $89.7 \%$ and $81.4 \%$ ). Sleep during the pandemic has been negatively affected in $72.6 \%$ of patients. Physical activity practice had significantly decreased during containment $(26.6 \%$ vs $51.7 \%)$

Conclusion: This study illustrates the social and psychological impact of COVID19 pandemic on patients with CIRD. In the light of those results, we have lunched an awareness campaign to reinforce psychologic and social support of patients with CIRD during this global health crisis.

Disclosure of Interests: None declared

DOI: 10.1136/annrheumdis-2021-eular.4184

\section{AB0902-HPR COVID-19 PANDEMIC: KNOWLEDGE, BELIEVES AND FEARS IN PATIENTS WITH CHRONIC INFLAMMATORY RHEUMATIC DISEASES IN A DEVELOPING COUNTRY}

T. El Joumani ${ }^{1}$, H. Rkain ${ }^{1,2}$, T. Fatima Zahrae ${ }^{1}$, H. Kenza ${ }^{3}$, A. Radouan ${ }^{4}$, N. Laila ${ }^{5}$, S. Bahloul ${ }^{1}$, E. A. Nada ${ }^{1}$, T. Latifa ${ }^{1}$, N. Hajjaj-Hassouni ${ }^{6}$, F. Allali ${ }^{1}{ }^{1}$ El Ayachi Hospital, Faculty of Medicine and Pharmacy of Rabat, Mohammed V University, Rabat, Morocco, Department of Rheumatology B, Rabat, Morocco; ${ }^{2}$ Faculty of Medicine and Pharmacy of Rabat, Mohammed V University, Rabat, Morocco, Laboratory of Physiology, Rabat, Morocco; ${ }^{3}$ Université Mohammed VI des Sciences de la Santé - UM6SS, Casablanca, Morocco., Ecole Internationale de Santé Publique, Rabat, Morocco; ${ }^{4}$ Faculty of Medicine and Pharmacy of Rabat, Mohammed V University, Rabat, Morocco, Laboratory of Biostatistics, Rabat, Morocco; ${ }^{5}$ Moroccan Association of Patients With Rheumatoid Arthritis and Spondyloarthritis, Rheumatology, Rabat, Morocco; ${ }^{6}$ International University of Rabat, Rabat, Morocco, Rheumatology, Rabat, Morocco

Objectives: To investigate the knowledge, believes, and fears of patients with Chronic Inflammatory Rheumatic Diseases (CIRD) in Morocco regarding the Covid-19 pandemic.

Methods: COVID-19 related knowledge was evaluated through following items: clinical presentations, age of infection, transmission routes, self-reported preventive behaviors, patient's approach in case of suspicion of being infected by COVID19 and vaccination. We also investigated the source of the patients' information.

Believes and fears of patients regarding COVID-19 was assessed using 2 items that explored participant's perception of the degree of threat to public health and their individual infection susceptibility compared to the general population. Results: A cross-sectional study was conducted among patients with rheumatic diseases using a questionnaire providing information on patients and disease characteristics. 350 patients had participated in this online survey (mean age of $46,1 \pm 14,4,68 \%$ were women, $15.7 \%$ were illiterate, $45.7 \%$ were unemployed, rural residence in $11.7 \%$ of cases). The correct answer rates of the 6 questions of the knowledge section of the questionnaire were $53.4-100 \%$ with an average mean of $90.7 \%$. The mean COVID-19 knowledge score was 5,43/6 (SD:0,48). Most participants (96.6\%) correctly identified the age group concerned about Covid-19 infection. Remarkably $92.3 \%$ of the participants recognized 3 clinical features when $97.7 \%$ and $72.3 \%$ of the sample recognized perfectly modes of transmission and disease prevention measures. $87.4 \%$ of patients knew the recommended course of action to do if they have fever or cough and have recently been in contact with an infected patient. $64 \%$ of the study population thought that there was no vaccine available yet.

The sources of information on COVID-19 were in descending order: rheumatologist $(56.6 \%$ ), personal research (on the internet or other) $(43.4 \%)$, video broadcasts on national television channels (28\%), other Awareness Video (24.2\%). Regarding the patients believes and fears toward COVID-19, $81.7 \%$ of patients believed that the pandemic is a serious threat for the public health and $80.6 \%$ thought that their CIRD makes them more exposed to the risk of contamination than general population.

Conclusion: The results of this survey show a high prevalence of patient concern about COVID-19. However, the majority of patients responded correctly on the mode of transmission and the means of protection for the COVID-19 infection. Those results could be explained by enormous awareness-raising efforts carried out by our differents departments of our government.

Disclosure of Interests: None declared

DOI: 10.1136/annrheumdis-2021-eular.4200

\section{HPR Service developments, innovation and eco- nomics in healthcare}

\section{AB0903-HPR DURATION OF TELEPHONE CONSULTATIONS AMONGST RHEUMATOLOGY PRACTITIONERS IN A DISTRICT GENERAL HOSPITAL - ADAPTING TO REMOTE CONSULTATION IN A COVID-19 PANDEMIC}

L. Parker ${ }^{1}$, F. Coldstream ${ }^{1} .{ }^{1}$ University Hospitals Dorset NHS Foundation Trust, Rheumatology Department, Christchurch, United Kingdom
Background: The Covid-19 pandemic has resulted in a rapid adoption of remote consultations in order to limit face to face clinical contact wherever appropriate, as recommended by the British Society for Rheumatology. The same clinic templates which existed for face-to-face encounters have been retrospectively adapted, without consideration of any potential difference in duration of consultations. Rheumatology practitioners from a variety of clinical backgrounds work alongside the rheumatology consultants, providing clinical care to patients with both inflammatory arthritis and connective tissue disease.

Objectives: To record the duration of all scheduled telephone consultations car ried out by advances rheumatology practitioners in a 4-week period.

Methods: All scheduled telephone clinic encounters over a 4-week period were timed and the duration recorded in a spreadsheet. Data was collected in real time by all 8 rheumatology advanced practitioners working within the rheumatology department of a district general hospital, following each clinic episode.

Results: Data was recorded from a total of 337 clinic appointments. Of these, $317(94 \%)$ were booked as routine, $3(0.9 \%)$ as urgent, $4(1.2 \%)$ were expedited following an advice line contact, and $13(3.9 \%)$ no data was recorded. $28(8 \%)$ of the patients did not answer when contacted. $80(24 \%)$ clinic appointments lasted 15 minutes or less, $186(55 \%)$ lasted 16 - 30 minutes, $37(11 \%)$ lasted 31 - 45 minutes, and $6(2 \%)$ lasted 46 - 60 minutes. The average duration was 22 minutes.

Conclusion: Within this department, remote consultations appear to have a similar duration when compared against the traditional clinic template for a fully faceto-face clinic, with some encounters lasting significantly longer than the planned duration. This would appear to differ to telephone consultations used in othe settings, such as general practice where the duration is reportedly shorter ${ }^{1}$. This may be representative of the additional complexity and co-morbidity of a typical rheumatology patient, or due to the multi-faceted nature of a rheumatology follow-up appointment ${ }^{2}$. Although remote consultations are effective in limiting risk of exposure to Covid-19, they may not offer a quicker or more efficient service compared with the face-to-face model. Further study in this field is required to evaluate this widely adopted new pattern of working.

\section{REFERENCES:}

[1] Pinnock H, Bawden R, Proctor S, Wolfe S, Scullion J, Price D, Sheikh A Accessibility, acceptability, and effectiveness in primary care of routine telephone review of asthma: pragmatic, randomised controlled trial. BMJ. 2003 Mar 1;326(7387):477-9. doi: 10.1136/bmj.326.7387.477. PMID: 12609944; PMCID: PMC150181.

[2] National Institute for Health and Care Excellence (NICE) (2018) rheumatoid arthritis in adults: management (NICE Guideline NG100). Available at https:// www.nice.org.uk/guidance/ng100 [Accessed 05 January 2021].

Disclosure of Interests: None declared

DOI: 10.1136/annrheumdis-2021-eular.1282

\section{AB0904-HPR RHEUMATOLOGY NURSING AND COVID19: FIRST WAVE EXPERIENCES}

L. Cano Garcia ${ }^{1}$, S. Garcia Diaz², S. P. Fernandez-Sanchez ${ }^{3}$, C. DomínguezQuesada $^{4}$, M. D. C. Ordoñez Cañizares ${ }^{1}$, J. M. Martín Martín ${ }^{5}$ on behalf of GTESER. ${ }^{1}$ Hospital Regional Universitario de Málaga, Rheumatology, Málaga, Spain; ${ }^{2}$ Hospital Moises Broggi, Rheumatology, Barcelona, Spain; ${ }^{3}$ Hospital de la Santa Creu i Sant Pau, Rheumatology, Barcelona, Spain; ${ }^{4}$ Hospital Universitario Virgen Macarena, Rheumatology, Sevilla, Spain $;{ }^{5}$ Hospital Universitario Virgen de la Candelaria, Rheumatology, Tenerife, Spain

Objectives: To know the impact of the first wave of COVID19 (March to June 2020) in the nursing activity in rheumatology and explore improvements in patient care during the pandemic situation.

Methods: 2 surveys were sent to nurses working in the rheumatology area (Both in the Outpatient department and day care unit) during October 2020 in Spain. The first survey had 10 questions about what happened in the rheumatology units in the first wave of the COVID19 pandemic (March to June of 2020) and the second survey had 10 questions about the standards of quality of nursing care in times of pandemic. Google forms were used to collect and analyze data.

Results: 32 nurses completed the survey (30 women (93.8\%)) with an average age of $49.6 \pm 10.2$ years. $93.8 \%$ work in the outpatient clinics and $2(6.2 \%)$ in day care units. The main results of the effects of the first wave of the pandemic are as follow: $4(12.5 \%)$ nurses were transferred to other areas of the hospital, $27(84.4 \%)$ showed changes in their care activity; and in $12(37,5 \%)$ of the surveyed centers, (slightly less than half of the medical staff) left their usual activity to care for covid-19 patients. In June 2020, at the end of the first wave, 17(53.1\%) reported more decompensated patients and $22(68.8 \%)$ reported that they had both telephone and face-to-face consultations; 15(46.9\%) reported that their clinical activity had increased.

Regarding the standards of quality during the pandemia: $27(84.4 \%)$ believed that rheumatology units should be strengthened,31(96.9\%) reported the need to carry out COVID educational campaigns in rheumatic patients and $30(100 \%)$ stated that nursing education should lead that education; 31(96.7\%) believed 